J. Clin. Chem. Clin. Biochem.

Vol. 15, 1977, pp. $345-348$

\title{
Zur Interaktion von Phenprocoumon mit Cyclamat
}

Von $A$. Kinawi und Ingrid Baumgartl

Aus dem Institut für Biochemie und Molekularbiologie am Fachbereich Biologie der Freien Universität Berlin

(Eingegangen am 1. Dezember 1976/23. Februar 1977)

Zusammenfassung: Der Einfluß von Cyclamat auf die gerinnungshemmende Aktivität von Phenprocoumon wurde an Ratten nach einmaliger oraler Applikation von Phenprocoumon, Cyclamat und Phenprocoumon/Cyclamat untersucht. Die zeitliche Änderung der Phenprocoumon-Konzentration im Serum wurde mit Hilfe der Hochdruckliquidchromatographie ermittelt und die gerinnungshemmende Wirkung von Phenprocoumon (Verlängerung der Prothrombinzeit, Quick-Test) bestimmt. Es ließ sich zeigen, daß Cyclamat eine Erhöhung der Konzentration von Phenprocoumon im Blut und eine Erniedrigung der gerinnungshemmenden Wirkung verursacht.

\section{Studies of the interactions of cyclamate with phenprocoumon}

Summary: The effect of cyclamate on the anticoagulant activity of phenprocoumon was investigated in rats after single oral doses of phenprocoumon, cyclamate, or phenprocoumon/cyclamate. By means of high pressure liquid chromatography [HPLC] the change of the concentration of phenprocoumon per unit time in sera was determined. The anticoagulant activity of phenprocoumon was determined by the extension of the prothromin-time (Quicktest). It was found that cyclamate gives rise to an elevated level of phenprocoumon in the blood, and a reduction of the anticoagulating potency.

\section{Einführung}

Die synthetischen Süßstoffe (Saccharin, Cyclamat bzw. eine Kombination beider $(1: 10)$ ) haben als Zuckerersatz in Nahrungsmitteln und Getränken bei medizinischer Indikation (Diabetes und Übergewicht) Eingang gefunden.

Die Literaturfülle der letzten fünf Jahre über Saccharin und insbesondere über Cyclamat dokumentiert das zunehmende wissenschaftliche Interesse auf diesem Gebiet. So sind zahlreiche Untersuchungen über die Toxizität (1-4), mögliche kanzerogene Wirkung (5-8), Biotransformation (9-12) und nicht zuletzt über die Analytik dieser Stoffe durchgeführt worden (13-16).

Während die Toxizität, die mögliche kanzerogene Wirkung, die Biotransformation und die Analytik den Schwerpunkt der Untersuchungen auf diesem Gebiet bilden, blieb die Frage der Inkompatibilität bzw. der Interaktion von Saccharin und Cyclamat mit anderen Arzneimitteln fast unbeachtet. Die wenigen Arbeiten, die sich mit der Interaktion bei gleichzeitiger Applikation von Cyclamat mit anderen Arzneistoffen beschäftigen, untersuchen lediglich den Einfluß dieser Arzneistoffe auf die Resorption und Biotransformation von Cyclamat. So untersuchten Ichibagase et al. und Suenaga et al. $(10,18,19)$ den Einfluß von Phenylbutazon, Phenobarbital, Tolbutamid, Neomycin und Sulfaguanidin auf den Stoffwechsel von Cyclamat (17-19). Im Rahmen unserer Untersuchungen über die Interaktion von oralen Antikoagulantien mit anderen Stoffen befassen wir uns in dieser Arbeit mit der Interaktion von Phenprocoumon mit Cyclamat.

\section{Material und Methoden}

\section{Versuchstiere}

Als Versuchstiere dienten 70 Ratten (B-DX SPF) beiderlei Geschlechts mit einem Körpergewicht von $260 \mathrm{~g}( \pm 5 \mathrm{~g})$. Sie erhielten Altro min-Trockenfutter und Leitungswasser ad libitum.

Die Versuchstiere wurden in vier Gruppen eingeteilt:

1. Gruppe (Phenprocoumon-Gruppe) 24 Ratten,

2. Gruppe (Phenprocoumon/Cyclamat-Gruppe) 24 Ratten,

3. Gruppe (Cyclamat-Gruppe) 16 Ratten und

4. Gruppe (Kontrollgruppe) 6 Ratten.

\section{Applikation}

Den Ratten wurde 11-12 h vor der Applikation nur das Futter entzogen. Die Applikation erfolgte im Ätherrausch einmalig oral (Gelatinekapsel Nr. 5, Parke-Davis und Co.). Die Versuchstiere der ersten Gruppe erhielten Phenprocoumon, die der zweiten 
Phenprocoumon und Cyclamat und die der dritten Cyclamat. Das Cyclamat lag als Natriumsalz vor. Bei der Applikation von Cyclamat und Phenprocoumon wurden beide Substanzen in eine Kapsel eingewogen. Die Dosis wurde stets gleich groß gehalten, sie lag bei je $38 \mathrm{mg} / \mathrm{kg}$ Körpergewicht. Nach erfolgreich verlaufener Applikation erhielten die Tiere wie üblich Futter und Wasser.

\section{Plasma- und Serumgewinnung}

Die Blutentnahme erfolgte $1,2,4,8,16,32,64$ und $128 \mathrm{~h}$ nach der Applikation (je drei Ratten von der ersten bzw. zweiten Gruppe und zwei von der dritten) unter Äthernarkose bei geöffnetem Thorax durch Herzpunktion. Zwecks Plasmagewinnung wurde jedem Versuchstier zuerst zweimal je $0,9 \mathrm{ml}$ Blut in je eine $1 \mathrm{ml}$ Plastikspritze, die je $0,1 \mathrm{ml}$ Natriumcitratlösung $(38 \mathrm{~g} / \mathrm{l})$ enthielt, entno mmen. Zur Serumgewinnung wurde der Rest an Blut in einer $10 \mathrm{ml}$-Plastikspritze aufgenommen. Von den nicht behandelten Ratten (4. Gruppe) wurde einer Ratte nach Versuchsbeginn alle $24 \mathrm{~h}$ auf gleiche Weise Blut entnommen (Blind-bzw. Normalwerte).

\section{Prothrombinzeitbestimmung}

Die so gewonnenen Plasmen dienten zur Prothrombinzeitbestimmung nach Quick (21). Die hierfür verwendeten SimplastinCalciumpräparate wurden von der Firma Labordiagnostica Gödecke (Hersteller: General-Diagnostics) bezogen. Die Bestimmung erfolgte nach Vorschrift des Herstellers.

\section{Phenprocoumonbestimmung}

\section{Extraktion der Seren}

Je 0,5 ml der erhaltenen Seren wurde mit je 5, 3 und schließlich $2 \mathrm{ml}$ Methylenchlorid extrahiert (Rüttler). Die Methylenchloridextrakte wurden portionsweise in einem Reagenzglas im Wasserbad bei $60^{\circ} \mathrm{C}$ bis zur Trockne eingedampft. Der Rückstand wurde erneut mit 3, 2 und schließlich $1 \mathrm{ml}$ Methylenchlorid (über Calciumchlorid getrocknet) extrahiert. Die einzeinen Extrakte wurden zur Entfernung von in trockenem Methylenchlorid unlöslichen Bestandteilen, die sich in der mobilen Phase wieder löser und eine Störung der quantitativen Bestimmung von Phen procoumon verursachen, mit Hilfe einer $5 \mathrm{ml}$-Spritze portionsweise durch eine mit Glaswolle gestopfte Kanüle in ein dickwandiges, spitz ausgezogenes kurzes Reagenzglas, welches im Wasserbad bei $60^{\circ} \mathrm{C}$ stand, gepreßt. Der auf diese Weise erhaltene trockene Extrakt wurde in 100-150 $\mu$ l der mobilen Phase (s. u.), die den internen Standard enthielt, bei $60^{\circ} \mathrm{C}$ in Lösung gebracht.

\section{Analytik}

Wie wir in anderen Arbeiten zeigen konnten (20, 22-25) ist die Hochdruckliquidchromatographie (HPLC) für die qualitative und quantitative Bestimmung von Pharmaka aus biologischem Material sehr gut geeignet. Im vorliegenden Fall erleichterte die Tatsache, daß Cyclamat in der hier anfallenden Konzentration keine Absorption zeigt, die Ermittlung des nachstehenden chromatographischen Systems.

Gerät: Varian 4800 Hochdruckliquid-Chromatograph.

Säule: $\quad$ Rostfreier Stahl, $183 \mathrm{~cm}, 2,1 \mathrm{~mm}$ Innendurchmesser.

Stationäre Phase: Merckosorb, SI 60, $10 \mu \mathrm{m}$.

Mobile Phase: Acetonitril/iso-Propanol $(100 \mathrm{ml}+20 \mathrm{ml})$ $70 \mathrm{ml} / \mathrm{h}$.

Detektor: UV bei $240 \mathrm{~nm}$ (UV-Vis-Spektralphotometer

Range:

Schreiber: $\quad$ Varian A 25, Spannung $50 \mathrm{mV}$, Papiervorschub

Interner-

Varian A 25, Spannung $50 \mathrm{mV}$, Papiervorsc
$50 \mathrm{~cm} / \mathrm{h}$. .

Standard-Lösung: $140 \mathrm{mg} / 1$ Koffein in der mobilen Phase.

Temperatur: $\quad 21-22^{\circ} \mathrm{C}$, angezeigter Druck: $325 \mathrm{~atm}$.

Bestimmung der Extraktionsausbeute von Phenprocoumon In 5 Reagenzgläser wurden $25,50,75,100$ bzw. $125 . \mu$ l einer methanolischen Phenprocoumonlösung $(200 \mathrm{mg} / \mathrm{l})$ pipettiert. Das Methanol wurde in Vakuum entfernt. Die Rückstände wurden mit je $1 \mathrm{ml}$ Serum (von nicht behandelten Ratten) versetzt und unter öfterem Rühren (Rüttler) $15 \mathrm{~min}$ im Wasserbad bei $37^{\circ} \mathrm{C}$ stehengelassen. Anschließend wurde, wie oben angegeben, extrahiert. Die Extrakte wurden auf ihren Phenprocoumongehalt analysiert.

\section{Ergebnisse}

Die Extraktionsausbeute für Phenprocoumon lag bei 97-98\% (20). Die Standardkurve für Phenprocoumon (Abb. 1) zeigt in dem hier interessierenden Konzentrationsbereich einen linearen Verlauf. Die quantitative Erfasșungṣgrenze für Phenprocoumon lag bei einer abso-

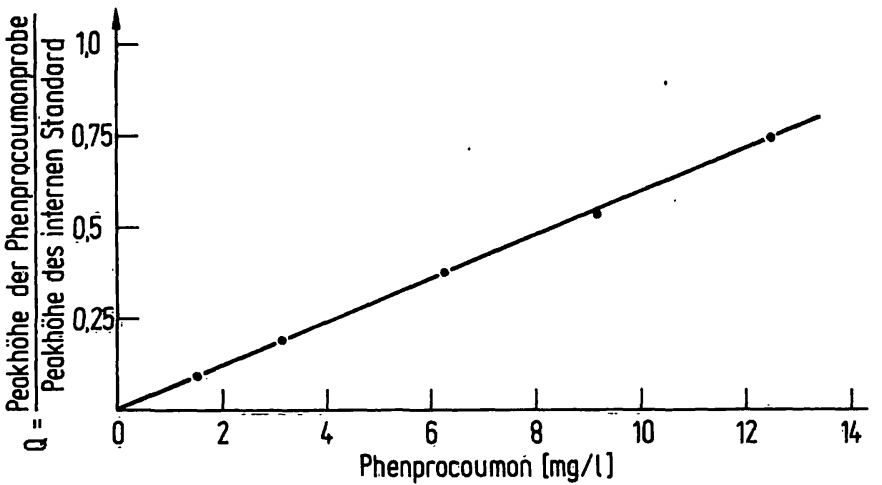

Abb. 1. Phenprocoumon-Standardkurve.

luten Peakhöhe von $10-12 \mathrm{~mm}$ bei etwa $5 \mu \mathrm{g}$ (gelöst in 5-50 $\mu$ der internen Standard-Lösung). Die HPLCAnalyse der Serumextrakte der unbehandelten Ratten (Blindprobe, Gruppe 4) zeigte, daß die quantitative Bestimmung von Phenprocoumon ungestört durchgeführt werden kann (keine Störpeaks, Abb. 2). Das gleiche Ergebnis zeigten ebenfalls die HPLC-Analyșen der Serumextrakte der Versuchstiere der 3. Gruppe. Der Normal-

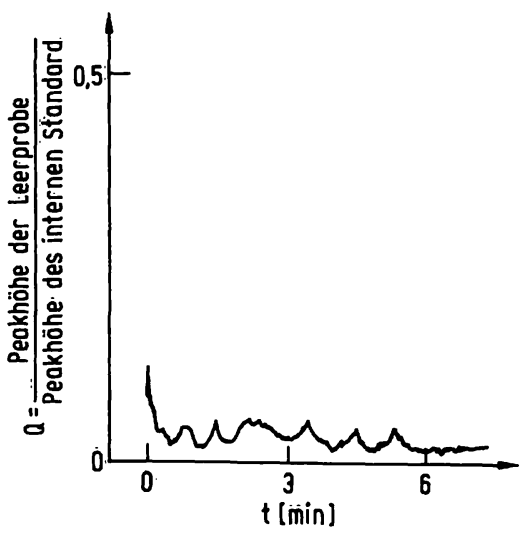

Abb. 2. HPLC-Analyse des Serumextraktes einer unbehandelten Ratte. Darstellung vgl. Abb. 5.

wert der Prothrombinzeit nach Quick lag bei $12 \mathrm{~s}$. Die ermittelten Mittelwerte der Konzentration von Phenprocoumon im Serum sowie die entsprechenden Prothrombinzeiten sind in der Abbildung 3 bzw. 4 graphisch dargestellt und in der Tabelle 1 wiedergegeben. 


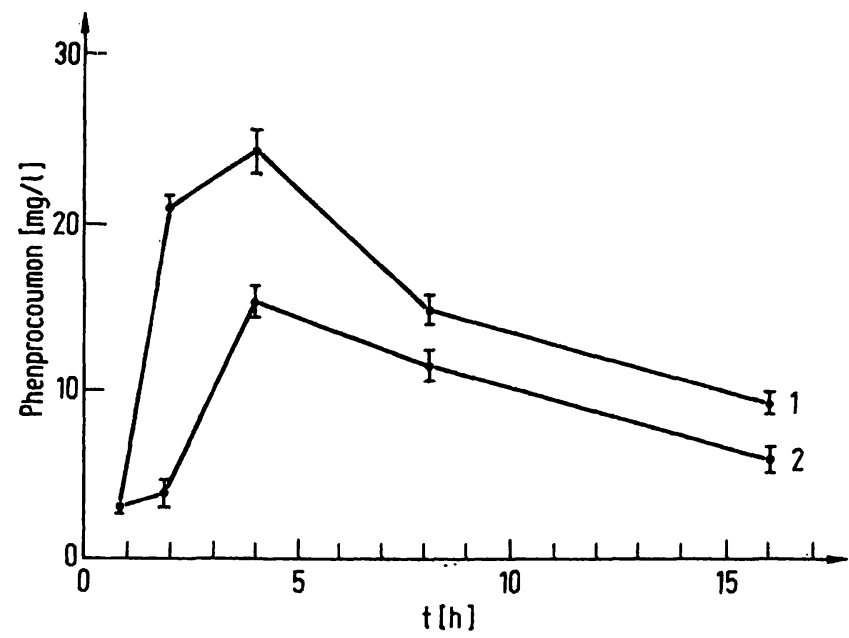

Abb. 3. Zeitliche Änderung der Konzentration von Phenprocoumon im Serum nach einmaliger oraler Applikation von

$1=$ Phenprocoumon/Natriumcyclamat

$2=$ Phenprocoumon

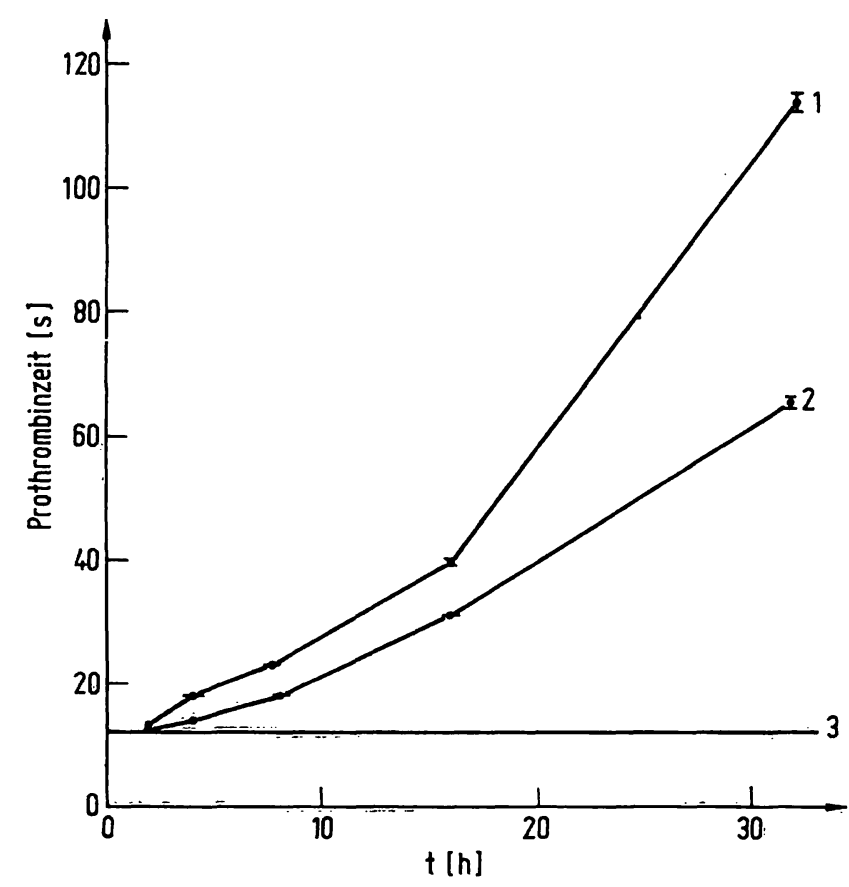

Abb. 4. Hemmung des Eiñflusses von Phenprocoumon auf die Blutgerinnung durch Cyclamat. Einmalige orale Applikation von

$1=$ Phenprocoumon

$2 \doteqdot$ Phenprocoumon/Natriumcyclamat

$3=$ Normalwert

Die in Abbildung 5 mit , ,2“ und , ,3“ beźifferten Peaks sind aüf hièr nicht näher untersuchte Stoffwechselprodukte zurückzuführen.

\section{Diskussion}

Die hohe Doșierung des Phenprocoumons ist aus mehreren Gründen erforderlich:

1. Phenprocoumon wurde nur einmalig oral appliziert.

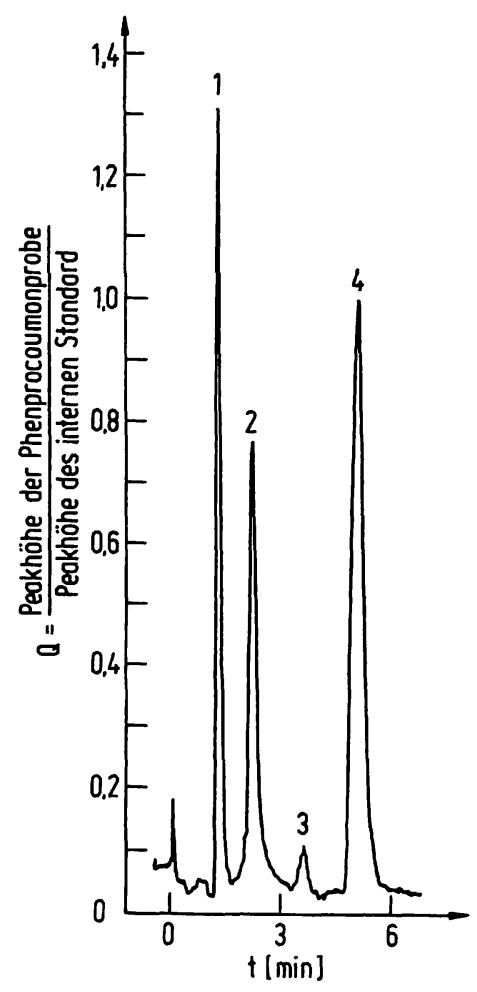

Abb. 5. HPLC-Analyse eines Serumextraktes $2 \mathrm{~h}$ nach oraler Applikation von Phenprocoumon/Natriumcyclamat. $1=$ Phenprocoumon

2 u. 3 = Metabolite

$4=$ interner Standard

Tab. 1. Mittelwerte der Phenprocoumon-Konzentration im Serum und zugehörige Prothrombinzeiten nach Applikation von Phenprocoumon allein bzw. Phenprocoumon und Cyclamat. Zu den angegebenen Zeiten wurden jeweils drei Versuchstiere (Doppelbestimmungen) untersucht.

\begin{tabular}{|c|c|c|c|c|}
\hline $\begin{array}{l}t \\
\text { (h) }\end{array}$ & $\begin{array}{l}\text { Phenproc } \\
\text { im Serum } \\
\text { nach App } \\
\text { von } \\
\text { Phenpro- } \\
\text { œumon } \\
\bar{x}\end{array}$ & $\begin{array}{l}\text { oumon } \\
\text { (mg/l) } \\
\text { likation } \\
\text { Phenpro- } \\
\text { coumon/ } \\
\text { Cyclamat } \\
\bar{x}\end{array}$ & $\begin{array}{l}\text { nach Appli } \\
\text { von } \\
\text { Phenpro- } \\
\text { coumon } \\
\bar{x}\end{array}$ & $\begin{array}{l}\text { Phenprocoumon/ } \\
\text { Cyclamat } \\
\overline{\mathbf{x}}\end{array}$ \\
\hline $\begin{array}{r}1 \\
2 \\
4 \\
8 \\
16 \\
32 \\
64\end{array}$ & $\begin{array}{r}3,31 \\
3,88 \\
15,25 \\
11,89 \\
6,43 \\
0,98 \\
0,83\end{array}$ & $\begin{array}{c}3,22 \\
22,22 \\
25,02 \\
14,92 \\
9,6 \\
2,18 \\
1,08\end{array}$ & $\begin{array}{l}12,0 \\
13,0 \\
18,0 \\
23,5 \\
39,5 \\
113,0 \\
\text { nicht zu } \\
\text { bestimmen } \\
>180 \\
\text { nicht zu } \\
\text { bestimmen } \\
>180\end{array}$ & $\begin{array}{l}12,0 \\
12,5 \\
13,5 \\
17,5 \\
31,5 \\
65,0 \\
\text { nicht zu } \\
\text { bestimmen } \\
>180 \\
\text { nicht zu } \\
\text { bestimmen } \\
>180\end{array}$ \\
\hline
\end{tabular}

2. Das Maximum der Phenprocoumonkonzentration im Serum liegt danach bei 15,25 bzw. $25,02 \mathrm{mg} / 1$. Dies entspricht $0,15 \%$ bzw. $0,25 \%$ der applizierten Menge in $1 \mathrm{ml}$ Serum. 
3. Die Reduzierung der Dosis (z. B. auf $1 / 10$ ) verursachte erhebliche analytische Schwierigkeiten, da in diesem Falle statt $0,5 \mathrm{ml}$ Serum $5 \mathrm{ml}$ extrahiert werden mußten. Da soviel Serum aus dem Blut einer Ratte nicht zu gewinnen ist, bleibt die Erhöhung des Volumens der auf die Säule injizierten Analysenprobe (50-80 $\mu$ statt 5-8 $\mu l)$ als Ausweg, der aber aus analytischen Gründen nicht wünschenswert ist (Überlastung der Säule, Bandenverbreiterung u. a. m.).

Die Mechanismen der Interaktionen mehrerer Pharmaka mit Antikoagulantien sind in wenigen Einzelfällen geklärt, wobei u. a. die Veränderung der Antikoagulantien-Plasmaeiweißbindung (Phenytoin, Phenylbutazon und Salicylate), Hemmung bzw. Aktivierung der Biotransformation von Antikoagulantien (Disulfiram, Barbiturate), Beeinflussung der Resorption aus dem Magen-Darm-Trakt (Antacida, Barbiturate) sowie die Änderung der Darmflora-Aktivität eine Rolle spielen $(26,27)$.

In dem hier vorliegenden Fall können mehrere dieser Ursachen eine Rolle spielen. Weiterhin läßt sich annehmen, daß Cyclamat einen Einfluß auf die Verteilung von Phenprocoumon im Organismus ausübt, wobei eine Anreicherung von Phenprocoumon im Blut erfolgt. Diese Erhöhung der Phenprocoumon-Konzentration im Blut kann eine Erniedrigung der Konzentration in der Leber, dem eigentlichen Zielorgan, zur Folge haben, wodurch die Synthese der Gerinnungsproteine entsprechend in geringerem Maße (verglichen mit der

\section{Literatur}

1. Taylor, J. D., Richards, R. K. \& Wiegand, R. G. (1968), Fd. Cosmet. Toxicol. 6, 313-327.

2. Friedman, L., Richardson, H. L., Richardson, M. E., Letho, E. J., Wallace, W. C. \& Sauro, F. M. (1972), J. Nat. Canc. Inst. 49, 751-764.

3. Barantom, P. G., Gaunt, I. F. \& Grasso, P. (1973), Fd. Cosmet. Toxicol. 11, 735-745.

4. Oser, B. L., Carson, S., Cox, G. E., Vogin, E. E. \& Sternberg, S. S. (1975), Toxicology 4, 315-330.

5. Schmähl, D. (1973), Arżneim.-Forsch. 23, 1466-1470.

6. Munro, I. C., Moodie, C. A., Krewski, D. \& Grice, H. C. (1975), Toxicol. Appl. Pharmacol. 32, 513-526.

7. Armstrong, B. \& Doll, R. (1974), Brit. J. Prev. Soc. Med. $28,233-240$.

8. Miller, R., White, L. S. \& Schwartz, H. J. (1974), J. Allergy Clin. Immunol. 53, 240-242.

9. Crampton, R. F. (1970), Br. Med. Bull. 26, 222-227.

10. Ichibagase, H., Kojima, S., Suenaga, A. \& Inoue, K. (1972), Chem. Pharm. Bull. 20, 1093-1101.

11. Wallace, W. C. \& Brouwer, E. H. (1970), J. Pharmacol. Exper. Therapeut. 175, 325-330.

12. Tesoriero, A. A. \& Roxon, J. J. (1975), Xenobiotica 5, 25-31.

13. Hoo, D. \& Hu, C. (1972), Anal. Chem. 44, 2111-2113.

14. Das, D. K., Mathew, T. V. \& Mitra, S. N. (1970), J. Chromatog. $52,354-356$.

15. Ratchik, E. M. \& Viswanathan, V. (1975), J. Pharmaceut. Sci. 64, 133-135.

16. Shenton, A: J. \& Johnson, R. M. (1973), Analyst 98 , 745-748. alleinigen Applikation von Phenprocoumon) gehemmt wird (s. Abb. 4). Die Annahme, daß die Änderung der Verteilung von Phenprocoumon im Organismus die Ursache für die Interaktion ist, läßt sich erhärten, wenn die hier erzielten Ergebnisse mit den Ergebnissen der unter der gleichen hier geschilderten Versuchsbedingung durchgeführten Untersuchung zur Interaktion von Chlordiazepoxid, Diazepam und Nitrazepam mit Phenprocoumon verglichen werden (20). Die Schwächung der gerinnungshemmenden Aktivität von Phenprocoumon durch die erwähnten Pharmaka-Wirkstoffe bzw. Cyclamat verhält sich umgekehrt proportional zu der Erhöhung der Phenprocoumon-Konzentration im Blut.

\section{Ob Cyclamat bzw. die Benzdiazepinderivate die} Phenprocoumon-Plasmaeiweißbindung verändern und wie diese Pharmakawirkstoffe bzw. Cyclamat bei mehrfacher Applikation mit Phenprocoumon dessen gerinnungshemmende Aktivität beeinflussen, ist Gegenstand noch nicht abgeschlossener Arbeiten.

\section{Danksagung}

Unser Dank gilt der Firma Hoffman-La Roche AG, GrenzachWyhlen und dem Fonds der Chemischen Industrie für Sachbeihilfen sowie Herrn $R$. Auste für die freundliche Unterstützung bei der Pflege der Versuchstiere.
17. Ichibagase, H., Kojima, S., Inoue, K. \& Suenaga, A. (1972), Chem. Pharm. Bull. 20, 175-180.

18. Ichibagase, H., Imamura, Y., Kinoshita, A. \& Kojima, S. (1972), Chem. Pharm. Bull. 20, 175-180.

19. Suenaga, A., Kojima, S. \& Ichibagase, H. (1972), Chem. Pharm. Bull. 20, 1357-1361.

20. Kinawi, A. \& Baumgartl, I. (1976), Arzneim.-Forsch. 26 , 2019-2023.

21. Quick, A. J., Stanly-Braun-M. \& Bancroft, F. W. (1935), Am. J. Med. Sci. 190, 501.

Noren, I. \& Quick, A. J. (1963), J. Lab. Med. 61, 302-305. Quick, A. J. (1963), Arch. Intern. Med. 111, 234-238.

22. Kinawi, A., Arzneim.-Forsch., im Druck.

23. Kinawi, A., Döring, B. \& Witte, I., Arzneim.-Forsch., im Druck.

24. Kinawi, A., Onken, A., Rozyczka, B. \& König, W., Arzneim.-Forsch., im Druck.

25. Kinawi, A. \& Schuster, T., Arzneim.-Forsch., im Druck.

26. Drug Interactions (Morselli, P., Cohen, S. N. \& Garattini, S. ed.), Raven Press, New York (1974).

27. Hansten, P. D. (1974), Arzneimittel-Interaktionen, Hippokrates Verlag, Stuttgart.
Ass. Prof. Dr. A. Kinawi Freie Úniversität Berlin Fachbereich Biologie WE 03

Ostpreußendamm 111

D-1000 Berlin 45 